\title{
The role of gut microbiota in immune checkpoint inhibitor therapy
}

\author{
Ming Yi, Shuang Qin, Qian Chu, Kongming Wu \\ Department of Oncology, the Tongji Hospital of Tongji Medical College, the Huazhong University of Science and Technology, Wuhan, 430030, \\ China \\ Correspondence to: Kongming Wu; Qian Chu. Department of Oncology, the Tongji Hospital of Tongji Medical College, the Huazhong University of \\ Science and Technology, Wuhan, 430030, China. Email: kmwu@tjh.tjmu.edu.cn; qianchu@tjh.tjmu.edu.cn. \\ Comment on: Routy B, Le Chatelier E, Derosa L, et al. Gut microbiome influences efficacy of PD-1-based immunotherapy against epithelial tumors. \\ Science 2018;359:91-7.
}

Submitted Sep 28, 2018. Accepted for publication Oct 10, 2018.

doi: 10.21037/hbsn.2018.11.12

View this article at: http://dx.doi.org/10.21037/hbsn.2018.11.12

It has been well established that the gut microbiota has a substantial influence on the host immune system. By crosstalk between specific microorganism-associated molecular patterns and the immune system, in addition to bacterial metabolic activity, gut microbiota regulates local or systemic inflammation (1). Recently, the composition of gut microbiota has been presumed to be one of the factors affecting the cancer-immune set point of cancer patients, which subsequently determines the efficacy of immune checkpoint inhibitor (ICI) treatment (2).

In a mouse xenograft model, Routy et al. noticed that the efficacy of anti-programmed death-1 ( $\alpha-\mathrm{PD}-1)$ or its combination with anti-cytotoxic T-lymphocyte associated protein 4 ( $\alpha$-CTLA-4) treatment was undermined, in the context of therapy, by broad-spectrum antibiotic or rearing in a specific pathogen-free (SPF) environment (3). A similar phenomenon was observed in non-small cell lung cancer (NSCLC) patients, and the main contributor to ICI resistance was speculated to be gut microbiota dysbiosis caused by broad-spectrum antibiotic. Further analysis of microbiota composition of patient fecal samples showed that some specific bacteria, such as A. muciniphila and Ruminococcus spp, were enriched in ICI responding patients (3). To confirm the enhanced treatment effect resulting from gut microbiota, 8 germ-free mice were prepared with fecal microbiota transplantation from different NSCLC patients (including 4 ICI responders and 4 ICI non-responders). After inoculation of MCA205 tumor cells, these mice received subsequent $\alpha$-PD1 treatment. As expected, tumor growth was suppressed in the mice with fecal microbiota transplantation from responders (3).

Given the unsatisfactory response rate of ICI (4), there is no doubt that this study meaningfully contributes to the understanding of how to overcome ICI resistance. In addition, other observations in the study confirmed the relationship between gut microbiota and ICI efficacy (Table 1). However, the exact mechanism by which gut microbiota influences the efficacy of ICI is still unclear. Presumably, multiple steps of the cancer-immunity cycle are promoted or inhibited by gut microbiota (Figure 1) $(10,11)$. The first step is cancer antigen presentation and $\mathrm{T}$ cell priming/activation. We observed that elevated abundance of Bifidobacterium was related with the upregulated transcription level of genes influencing production of cytokines (e.g., interferon- $\gamma$ ) in dendritic cells (DC) (5). DC is one of the most important antigen presentation cells (APC) and initiates the whole antitumor immune response. The second step of the cancerimmunity cycle is the trafficking and infiltration of $\mathrm{T}$ cells into a tumor condition. We further found that recolonization of $A$. muciniphila and $E$. birae in germ-free mice was followed by the emergence of $\mathrm{CXCR}^{+}{ }^{+} \mathrm{CCR}^{+}$ central memory $\mathrm{T}$ cells $\left(\mathrm{T}_{\mathrm{CM}}\right)$ in the tumor draining lymph node and tumor bed, indicating that more $\mathrm{T}$ cells were recruited to the tumor (3). The last step of the cycle is the recognition of cancer antigen and cytotoxic effect by the $\mathrm{T}$ cells. We discovered that cancer neoantigen was homological with some non-self materials from the microbiota which indicates the potential molecular mimicry between the microbiota and cancer cells (12). Release of cancer neoantigen in vivo could have enhanced 
Table 1 Influence of gut microbiota on ICI treatment

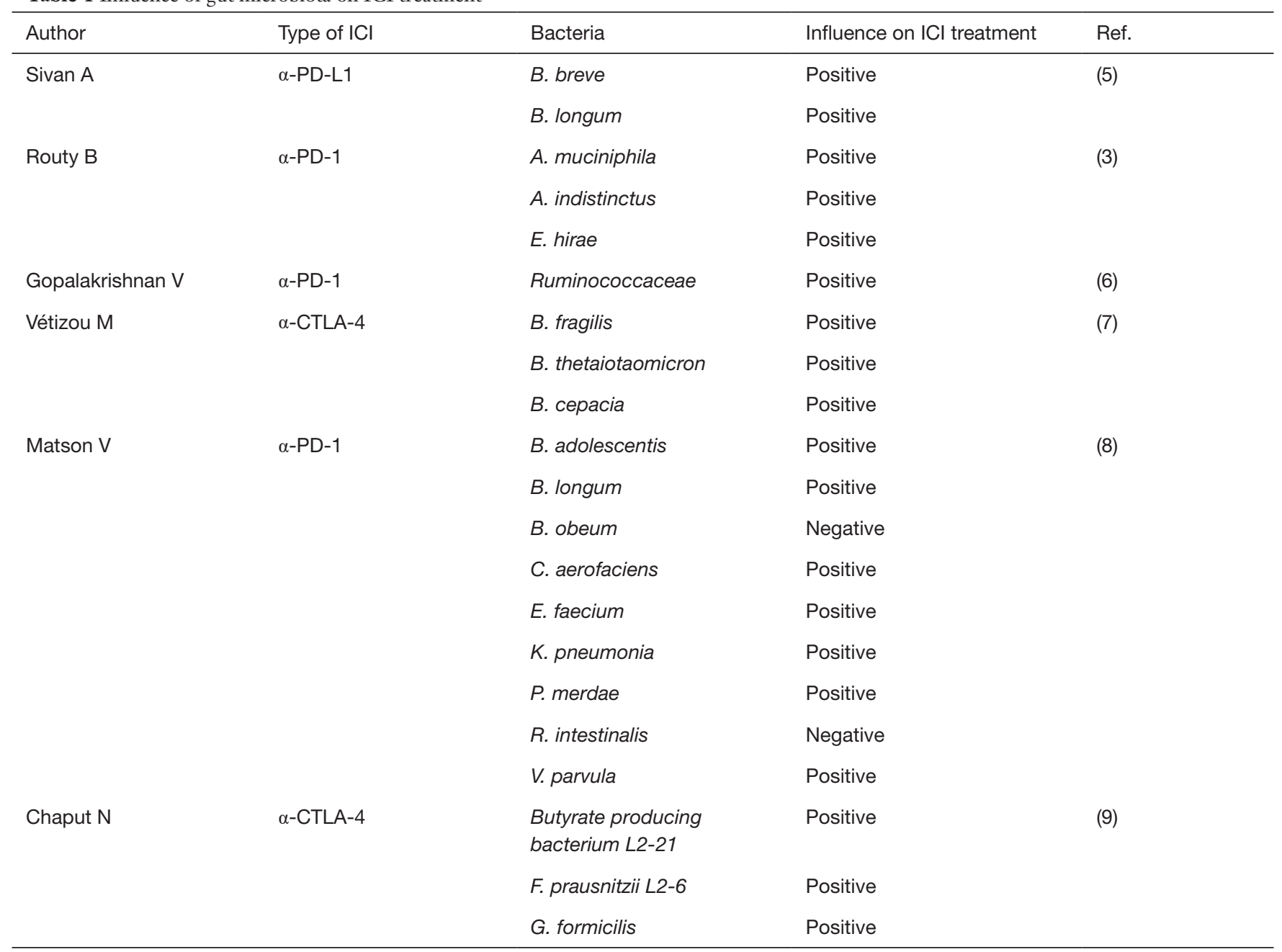

$\alpha$-CTLA-4, anti-cytotoxic T-lymphocyte associated protein 4; $\alpha$-PD-1, anti- $\alpha$-programmed death-1; $\alpha$-PD-L1, anti-programmed death-ligand 1; $\mathrm{ICl}$, immune checkpoint inhibitor.

tumor-specific activity by both a broadened T-cell receptor (TCR) spectrum and increased $\mathrm{T}$ cell abundance. In addition to cross-reactivity, regulatory $\mathrm{T}$ cell (Treg) is another factor determining $\mathrm{T}$ cell activity. Faecalibacterium prausnitzii and Clostridia promote Treg differentiation and increase the size of the Treg pool (13). Meanwhile, Treg is the primary target of $\alpha$-CTLA- 4 therapy which means these bacteria might be predictors for $\alpha$-CTLA- 4 therapy.

The findings of Routy et al. reveal that the composition of the gut microbiota is a predictive biomarker and treatment target in ICI therapy. Also, fecal microbiota transplantation is believed to be a promising approach to enhance ICI efficacy and overcome resistance. However, further investigation is needed for the application of gut microbiota in clinical practice. Previous studies have analyzed gut microbiota using a fecal sample; however, Zmora et al. confirmed that microbiota obtained from stool was not a perfect surrogate of gut mucosa-associated microbiota (14). Partial correlation between fecal microbiota and gut mucosa-associated microbiota might result in the misinterpretation of gut microbiota. Moreover, the colonization of transplanted microbiota might be interfered with by the indigenous microbiota. Indeed, the resistance to colonization of transplanted microbiota varies from person to person, suggesting that a personalized strategy is essential to the proper application of gut microbiota (14). 


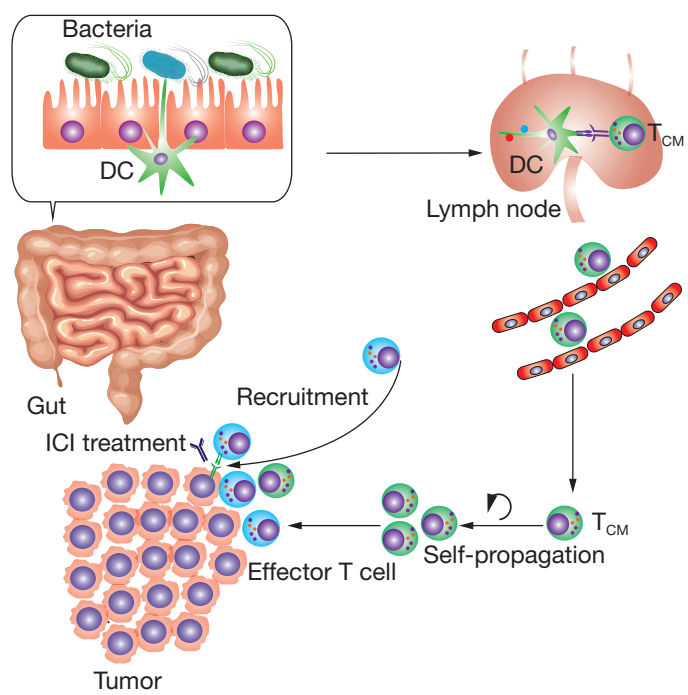

Figure 1 Main mechanism by which the gut microbiota influences ICI treatment. The homology between non-self material of the gut microbiota and tumor antigen induces cross-reactivity of $\mathrm{T}$ cells. Dendritic cells (DC) recognize the non-self material of gut microbiota, then present the antigen and activate the $\mathrm{T}$ cells in the lymph node. Exposure to antigens and central memory $\mathrm{T}$ cells $\left(T_{C M}\right)$ is formed. Subsequently, accumulated TCM in tumor bed could differentiate to effector $\mathrm{T}$ cell and further recruit effector $\mathrm{T}$ cells from peripheral circulation. The increased $\mathrm{T}$ cell activity has a positive influence on immune checkpoint inhibitor (ICI) treatment.

\section{Acknowledgements}

We thank Dr. Tianye Li for the language editing assistance. Funding: This work was supported by the National Natural Science Foundation of China (No. 81572608, 81874120, 81672984), and the Wuhan Science and Technology Bureau (No. 2017060201010170).

\section{Footnote}

Conflicts of Interest: The authors have no conflicts of interest to declare.

\section{References}

1. Cani PD, Jordan BF. Gut microbiota-mediated inflammation in obesity: a link with gastrointestinal cancer. Nat Rev Gastroenterol Hepatol 2018;15:671-82.

2. Chen DS, Mellman I. Elements of cancer immunity and the cancer-immune set point. Nature 2017;541:321-30.
3. Routy B, Le Chatelier E, Derosa L, et al. Gut microbiome influences efficacy of PD-1-based immunotherapy against epithelial tumors. Science 2018;359:91-7.

4. Li X, Shao C, Shi Y, et al. Lessons learned from the blockade of immune checkpoints in cancer immunotherapy. J Hematol Oncol 2018;11:31.

5. Sivan A, Corrales L, Hubert N, et al. Commensal Bifidobacterium promotes antitumor immunity and facilitates anti-PD-L1 efficacy. Science 2015;350:1084-9.

6. Gopalakrishnan V, Spencer CN, Nezi L, et al. Gut microbiome modulates response to anti-PD-1 immunotherapy in melanoma patients. Science 2018;359:97-103.

7. Vétizou M, Pitt JM, Daillere R, et al. Anticancer immunotherapy by CTLA-4 blockade relies on the gut microbiota. Science 2015;350:1079-84.

8. Matson V, Fessler J, Bao R, et al. The commensal microbiome is associated with anti-PD-1 efficacy in metastatic melanoma patients. Science 2018;359:104-8.

9. Chaput N, Lepage P, Coutzac C, et al. Baseline gut microbiota predicts clinical response and colitis in metastatic melanoma patients treated with ipilimumab. Ann Oncol 2017;28:1368-79.

10. Chen DS, Mellman I. Oncology meets immunology: the cancer-immunity cycle. Immunity 2013;39:1-10.

11. Yi M, Yu S, Qin S, et al. Gut microbiome modulates efficacy of immune checkpoint inhibitors. J Hematol Oncol 2018;11:47.

12. Kim S, Kim HS, Kim E, et al. Neopepsee: accurate genome-level prediction of neoantigens by harnessing sequence and amino acid immunogenicity information. Ann Oncol 2018;29:1030-6.

13. Furusawa Y, Obata Y, Fukuda S, et al. Commensal microbe-derived butyrate induces the differentiation of colonic regulatory T cells. Nature 2013;504:446-50.

14. Zmora N, Zilberman-Schapira G, Suez J, et al. Personalized Gut Mucosal Colonization Resistance to Empiric Probiotics Is Associated with Unique Host and Microbiome Features. Cell 2018;174:1388-405.e21.

(English Language Editor: John Ayric Gray, AME Publishing Company)

Cite this article as: Yi M, Qin S, Chu Q, Wu K. The role of gut microbiota in immune checkpoint inhibitor therapy. HepatoBiliary Surg Nutr 2018;7(6):481-483. doi: 10.21037/ hbsn.2018.11.12 